\title{
Reinfection in American Cutaneous Leishmaniasis: Evaluation of Clinical Outcomes in the Hamster Model
}

\author{
Y Osorio/ ${ }^{+}$, SJ Gonzalez*, VL Gama*, BL Travi \\ Centro Internacional de Entrenamiento e Investigaciones Médicas (CIDEIM), AA 5390, Cali, Colombia \\ *Universidad de Los Andes, Santafé de Bogotá, Colombia
}

\begin{abstract}
There is no clear understanding of the outcome of reinfection in New World cutaneous leishmaniasis, and its role in the relationship to the development of protection or secondary disease. For this reason, reinfection experiments with homologous (Leishmania panamensis-L. panamensis) and heterologous (L. major-L. panamensis) species of leishmaniae were conducted in the hamster model. The different protocols for primary infections prior to the challenge with L. panamensis were as follows: $(a)$ L. major, single promastigote injection, (b) L. major, three booster infections, (c) L. panamensis, followed by antimonial treatment to achieve subclinical infection, $(d) \mathrm{L}$. panamensis, with active lesions, (e) sham infected, naive controls. Although all reinfected hamsters developed lesions upon challenge, animals with active primary lesions due to L. panamensis, and receiving booster infections of L. major had the most benign secondary lesions (58-91\% and 69-76\% smaller than controls, respectively, $P<0.05$ ). Subclinically infected animals had intermediate lesions (40-64\% smaller than controls, $P<0.05)$, while hamsters which received a single dose of L. major had no significant improvement over controls. Our results suggested that $\mathrm{L}$. major could elicit a cross protective response to $\mathrm{L}$. panamensis, and that the presence and number of amastigotes persisting after a primary infection may influence the clinical outcome of reinfections.
\end{abstract}

Key words: immunoprophylaxis - reinfection - Leishmania major - Leishmania panamensis - hamster

Human populations in leishmaniasis endemic areas are continuously exposed to the bites of sandflies and consequently are at risk of suffering repeated infections (Sebai \& Morsy 1976, Weigle et al. 1993). Understanding the clinical and immunological responses of individuals who are reinfected could shed some light on the most adequate approaches for adopting prevention and control strategies.

In the Old World, leishmanization with small doses of Leishmania major in body sites usually covered by clothes was used as an immunoprophylactic strategy. Although this method resulted in total or partial protection against a subsequent infection, leishmanization has been abandoned due to the risk of occasional severe lesions, and the fact that a live virulent organism was introduced into humans (Greenblatt 1988, Modabber 1990).

\footnotetext{
This work was supported by the U.S. National Institute of Allergy and Infectious Diseases, National Institutes of Health, Tropical Medicine Research Center Grant P50AI30603-04 and COLCIENCIAS 2229-04-004-92.

+Corresponding author. Fax: +57-2-667.2989

Received 15 August 1997

Accepted 18 February 1998
}

In the New World, the frequency of reinfections is more difficult to assess because a variable proportion of individuals may relapse or suffer mucosal metastasis after a primary infection (Marsden 1986). The higher incidence of cutaneous leishmaniasis in children (CIDEIM, unpublished data) and young adults of the Colombian Pacific coast (Weigle et al. 1993), suggests that older individuals have developed an effective immune response against the parasite. Nevertheless, the proportion of exposed people capable of mounting a protective response against reinfection has yet to be determined. In a limited study involving 24 patients with secondary lesions due to L. Viannia spp. it was shown, through kDNA restriction fragment electrophoresis and molecular karyotyping, that reinfection accounted for $50 \%$ of the cases, implying that these individuals had not developed a protective immune response as a consequence of the primary infection (Saravia et al. 1990).

Experimental data about reinfection with the viannia subgenus are scarce, mainly due to the lack of adequate laboratory models in which immunological responses could be studied. The contrasting results of reinfection obtained in $\mathrm{C} 57 \mathrm{~B} 1 / 6$ mice infected with L. mexicana (Pérez et al. 1979, Aragort et al. 1993), as well as the inconsistent protective responses observed within each species in Mystromys albicaudatus and Callithrix 
penicillata (Beacham et al. 1982, Cuba-Cuba \& Marsden 1993), underscore the need for a systematic approach to this subject. In order to evaluate the utility of the hamster as a model for immunoprophylactic strategies against $L$. Viannia spp. we carried out reinfection experiments with L. major and L. panamensis.

\section{MATERIALS AND METHODS}

A subline of inbred golden hamsters (Chester Beaty, Charles River Laboratory, Wilmington, Ma) was used in all the experiments. To evaluate the effect of a heterologous reinfection 12 hamsters were inoculated intradermally (i.d.) in the right hind foot with $1 \times 10^{6}$ promastigotes of $L$. major (MHOM/SV/73/5ASKH) harvested from the logarithmic phase of growth in Schneider's Drosophila medium (GIBCO). Two booster infections at 15day intervals were applied in a similar manner to six of these hamsters. Two weeks later the groups, which receive single or multiple inocula, together with a group of naive animals $(n=6)$, were subjected to a challenge infection in the right forefoot with $1 \times 10^{4}$ stationary phase promastigotes of $L$. panamensis (MHOM/COL/83/2058).

The effect of a homologous reinfection was determined by inoculating $1 \times 10^{6}$ stationary phase promastigotes of L. panamensis (MHOM/COL/84/ 1099) i.d. in the hindfoot of 12 hamsters. Twenty days p.i. one subgroup of animals $(n=6)$ was treated with a subcurative dose of antimonials, which in the particular case of this batch of Glucantime ${ }^{\circledR}$ (Specia-Rhone Poulenc) was $150 / \mathrm{mg} / \mathrm{kg} /$ day for 20 days. Persistent infection was confirmed by the lack of conspicuous lesions (foot diameter $<0.5$ $\mathrm{mm}$ as compared with controls), and by positive aspirate-culture of the inoculation site. Hamsters with subclinical infections or with active lesions, and naive animals $(n=6)$ were challenged i.d. in the forefoot with $1 \times 10^{4}$ stationary phase promastigotes of a different $L$. panamensis strain (MHOM/COL/83/2058).

Lesion evolution in anethesized hamsters (ketamine hidrochloride 50mg/kg, Parke Davis + $20 \mathrm{mg} / \mathrm{kg}$ xylacine, Bayer) was monitored every seven days until the 5th week post-challenge by measuring the diameter of the foot with a digital caliper (Mitutoyo Corporation, Japan). Data were analyzed by ANOVA and t- test.

Animals were sacrificed three months postinfection, and their parasitological status determined by culturing samples from the site of primary and secondary infection, including the regional lymph node. Cultures were incubated at $25^{\circ} \mathrm{C}$ for up to one month, and subjected to weekly inspections. Persistence and distribution of the different strains used in the inoculations, was deter- mined by IFAT with the aid of monoclonal antibody B3 (XLV-5B8) specific for L. major, and B4 (VI-2A5A4) and B11 (VII-5G3F3) both specific for $L$. panamensis.

\section{RESULTS}

Hamsters subjected to a single infective injection of L. major did not develop primary lesions $(0.2 \mathrm{~mm} \pm 0.1)$, while those subjected to booster infections showed lesions of moderate size $(1 \mathrm{~mm}$ \pm 0.4 ) with central necrosis that self-healed at ten weeks. Not significant differences with controls were observed in the size of lesions after two weeks post-challenge with $L$. panamensis in individuals that received a single primary inoculum with $L$. major (Fig. 1). On the other hand, hamsters subjected to booster infections with L. major showed smaller lesions upon challenge than control individuals (Fig. 2), and non-quantitative inflammatory signs such as redness and depilation also were less conspicuous. Differences in lesion size varied with time of evolution, being 69 to $76 \%$ smaller than in controls $(\mathrm{P}<0.05)$. By means of aspirateculture and IFAT using monoclonal antibodies, $L$. major was detected only in two of the six hamsters, one at the inoculation site (1/6) and the other in the regional lymph node (1/6). On the other hand, L. panamensis was detected not only at the site of challenge in the forefoot (6/6), but also in the lymph node where the primary infection with $L$. major was accomplished (2/6). It was not possible to recover L. major from the tissues of hamsters which received a single inoculum of this parasite.

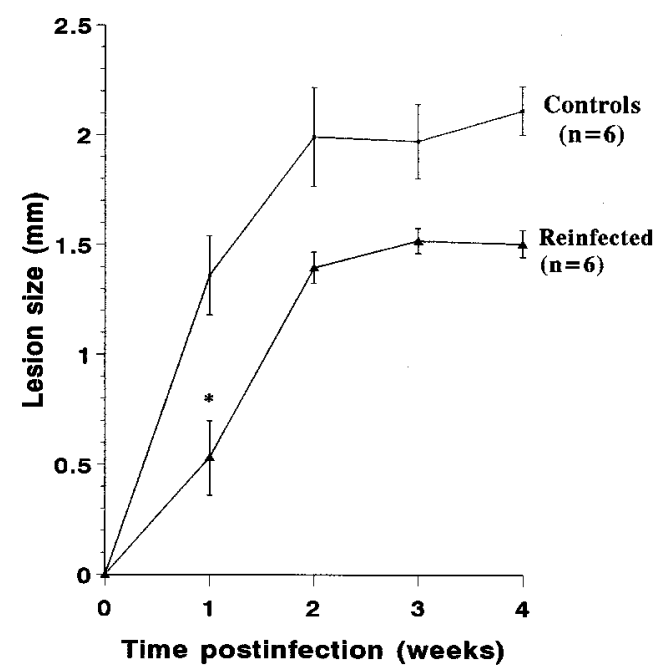

Fig. 1: evolution of lesions (mean $\pm \mathrm{SE}$ ) at the site of challenge in hamsters reinfected with Leishmania panamensis, that were previously infected with a single infection of $L$. major as compared with naive controls $(* \mathrm{P}=0.05)$. 


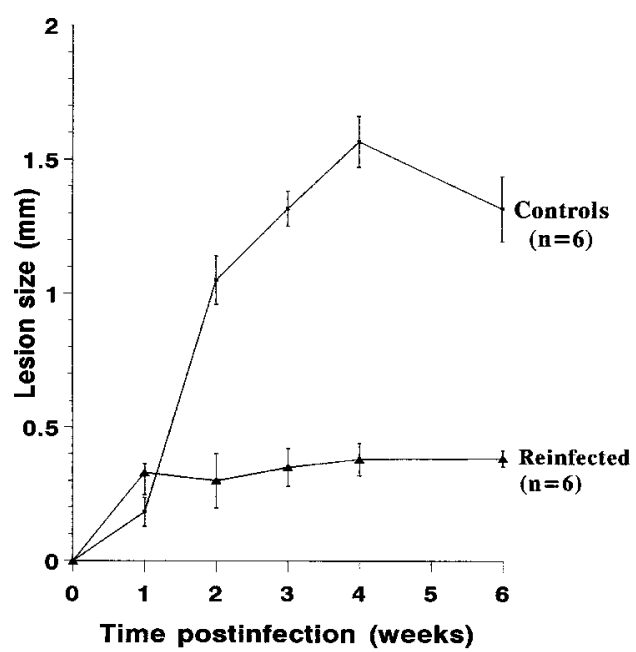

Fig. 2: evolution of lesions (mean $\pm \mathrm{SE}$ ) at the site of challenge in hamsters reinfected with Leishmania panamensis, that were previously infected with three booster infections of L. major as compared with naive controls.

Subclinical infection in Glucantime ${ }^{\circledR}$ treated hamsters was confirmed by aspirate-culture from the inoculation site (6/6) and the regional lymph node $(3 / 6)$. Two weeks after the challenge infection with $L$. panamensis in the forefoot, the resulting lesions were 40-64\% smaller than in control animals $(\mathrm{P}<0.05$; Fig. 3$)$. The group of hamsters with active primary lesions at the time of challenge showed the greatest difference compared with controls as regards lesion size produced by the challenge infection. In these animals the lesions induced by reinfection were $58-91 \%$ smaller than in controls $(\mathrm{P}<0.05)$, and $28-76 \%$ smaller than in subclinically infected individuals $(\mathrm{P}<0.05$; Fig. 3$)$.

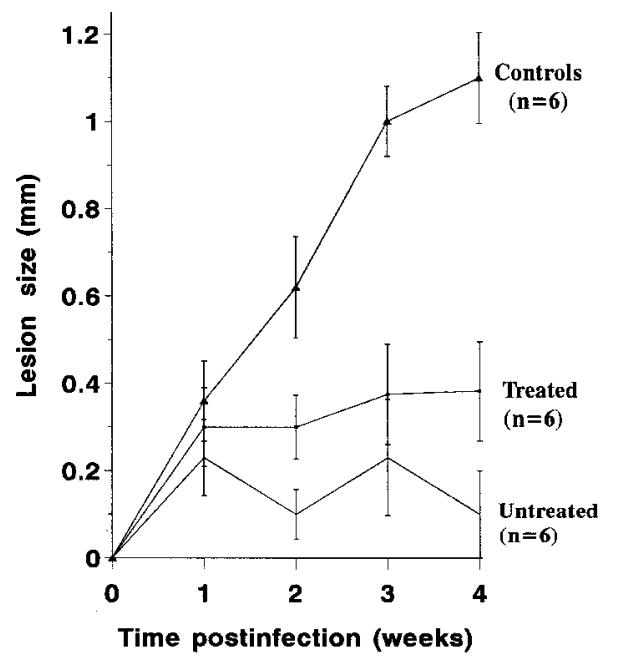

Fig. 3: evolution of lesions (mean $\pm \mathrm{SE}$ ) at the site of challenge in hamsters infected and reinfected with Leishmania panamensis, with active primary lesions (untreated) or subclinical infection (treated), as compared with naive controls.

\section{DISCUSSION}

These experiments showed that hamsters are capable of mounting a partially protective immune response against $L$. Viannia spp., which was clinically evident through the development of smaller lesions upon reinfection. A similar response has been observed in patients from the New World (L. Viannia spp.) and Old World (L. major) in whom secondary lesions were consistently smaller than primary lesions (Bienzle et al. 1978, Saravia et al. 1990).

The partial cross-protection achieved against $L$. panamensis using boosting injections of $L$. major as a primary inoculum, suggested that this Leishmania species could have some immunoprophylactic potential in the New World. However, there is an anecdotal report of a susceptible patient who suffered a previous episode of cutaneous leishmaniasis due to L. braziliensis that developed lesions upon two subsequent infections with $L$. major (Killick-Kendrick et al. 1985).

The route of inoculation, which may have a different effect upon the host immune response, has not been well defined either in humans or animal models. In fact, results could vary from one laboratory animal to the other. In our study the intradermal route led to the partial protection of hamsters, while an exacerbating effect has been observed in the mouse-L. major system (Mitchell et al. 1981).

The isolation of L. major or L. panamensis from the healed lesions of hamsters is consistent with previous observations regarding parasite persistence concomitant with a partially effective immune response, both in humans and laboratory animals (Rivier et al. 1993, Aebischer 1994). It is worth noting that in our experiment no L. major could be detected in the animals that received a single injection of live promastigotes, and were unprotected upon challenge with L. panamensis.

The significant amelioration in the size of secondary lesions in hamsters with active primary lesions as compared with subcuratively treated animals, suggests that the immune response could vary according with the parasite load existent at the time of challenge. In fact, other authors have hypothesized that the presence of antigen could be necessary for maintaining $\mathrm{T}$ cell memory (Moll et al. 1995). This hypothesis is supported by the progressive loss of an effective immune response in mice infected with $L$. arabica and subsequently reinfected with $L$. major, which was negatively correlated with the time interval between primary and challenge injections (Neal et al. 1990). Under the light of these results, the convenience of a live attenuated vaccine which implies persistence of the parasite in the host, versus putatively safer short 
lasting killed vaccines should be carefully evaluated in preclinical trials.

The present study showed that the hamster could be used as an adequate model for understanding the factors that participate in the clinical response to L. Viannia. Most importantly, to understand whether elimination of parasites or subclinical infections are necessary for developing and sustaining a protective immune response, and from a more practical standpoint, the effect of booster infections, and routes of administration in immunoprophylactic approaches.

\section{REFERENCES}

Aebischer T 1994. Recurrent cutaneous leishmaniasis: A role for persistent parasites? Parasitol Today 10: 25-28.

Aragort R, Rossell O, De Jesús R, Rodriguez AM 1993. Thermotherapy v. antimonials: what happens after the healing of cutaneous leishmaniasis lesions? Ann Trop Med Parasitol 87: 23-30.

Beacham B, Romito R, Kay D 1982.Vaccination of the african white-tailed rat, Mystromys albacaudatus, with sonicated Leishmania braziliensis panamensis promastigotes. Am J Trop Med Hyg 31: 252-258.

Bienzle U, Ebert F, Dietrich M 1978. Cutaneous leishmaniasis in Eastern Saudi Arabia. Epidemiological and clinical features in a non-immune population living in an endemic area. Tropen Med Parasitol 29:188-193.

Cuba-Cuba CA, Marsden PD 1993. Marmosets in New World leishmaniasis research. Medicina (Buenos Aires) 53: 419-423.

Greenblatt CL 1988. Cutaneous leishmaniasis: the prospects for a killed vaccine. Parasitol Today 4: 53-54.

Killick-Kendrick R, Bryceson ADM, Peters W, Evans DA, Leaney AJ, Rioux JA 1985. Zoonotic cutaneous leishmaniasis in Saudi Arabia: lesions healing naturally in man followed by second infection with the same zymodeme of Leishmania major. Trans $R$ Soc Trop Med Hyg 79: 363-355.

Marsden PD 1986. Mucosal leishmaniasis ("espundia" Escomel, 1911). Trans R Soc Trop Med Hyg 80: 859876.

Mitchell GF, Curtis JM, Hanman E 1981. Resistance to cutaneous leishmaniasis in genetically susceptible BALB/c mice. Aust J Exp Biol Med Sc 59: 555.

Modabber F 1990. Development of vaccines against leishmaniasis. Scand J Infect Dis 76: 72-78.

Moll H, Flohe S, Rollinghoff M 1995. Dendritic cells in Leishmania major immune mice harbor persistent parasites and mediate an antigen-specific T cell immune response. Eur J Imm 25: 693-699.

Neal RA, Reeves A, Peters W 1990. Leishmania infecting man and wild animals in Saudi Arabia 7. Partial protection of mice against Leishmania major by prior infection with $L$. arabica. Trans $R$ Soc Trop Med Hyg 84: 233-238.

Pérez H, Arredondo B, Machado R 1979. Leishmania mexicana and Leishmania tropica: cross immunity in C57BL/6 mice. Exp Parasitol 48: 9-14.

Rivier D, Shah R, Bovay P, Mauel J 1993. Vaccine development against cutaneous leishmaniasis. Subcutaneous administration of radioattenuated parasites protects CBA mice against virulent Leishmania major challenge. Parasite Immunol 15: 75-84.

Saravia NG, Weigle K, Segura I, Holmes S, Pacheco R, Labrada LA, Gonçalves A 1990. Recurrent lesions in human Leishmania braziliensis infection-reactivation or reinfection? The Lancet 336: 398-402.

Sebai ZA, Morsy TA 1976. Cutaneous leishmaniasis in Bisha Town, Saudi Arabia. J Trop Med Hyg 79: 8991.

Weigle KA, Santrich C, Martinez F, Valderrama L, Saravia NG 1993. Epidemiology of cutaneous leishmaniasis in Colombia: a longitudinal study of the natural history, prevalence, and incidence of infection and clinical manifestations. J Inf Dis 168: 699708. 